\title{
RÉPLICA
}

\section{REPENSANDO GUATEMALA EN LA ÉPOCA DE RAFAEL CARRERA. RESPUESTA A GUSTAVO PALMA MURGA ${ }^{1}$

\author{
Brian Connaughton \\ Universidad Autónoma Metropolitana-Iztapalapa
}

"Una razón clara y convincente puede influir a la mente, tanto de un oyente erudito como ignorante, mientras viva...”

"La pasión nunca debe prevalecer sobre la razón."

"Razonar jamás hará que un hombre corrija una opinión equivocada, que por razonamiento nunca adquirió...”.2

${ }^{1}$ Gustavo Palma Murga, Sobre Brian Connaughton (coord.), Repensando Guatemala en la época de Rafael Carrera. El país, el hombre y las coordenadas de su tiempo, México, Gedisa, Universidad Autónoma Metropolitana-Iztapalapa, 2015, 528 pp. ISBN 978-84-9784-986-9 (Gedisa), ISBN 978-60728-0432-6 (UAM); Historia Mexicana, LxIx: 4 (276) (abr.-mayo 2020), ISSN 2448-6531 DOI: http://dx.doi.org/10.24201/hm.v69i4.3720

2 [Jonathan Swift], A Letter to a Young Gentleman, Lately Enter'd Into Holy Orders by a Person of Quality, Second Edition, Londres, Printed for J. Roberts at the Oxford Arms in Warwick Lane, 1721, carta original fechada el 9 de enero de 1720, pp. 14, 27 y 29: “A plain convincing Reason may possibly operate upon the Mind both of a learned and ignorant Hearer as long as they live..."; "Passion should never prevail over Reason"; "Reasoning well never make a Man correct an ill Opinion, which by Reasoning he never acquired...". 
F lautor de esta reseña, Gustavo Palma Murga, trató con alguC na seriedad sólo tres estudios de la obra, que al parecer fueron de su agrado. Brincó enteramente una amplia introducción que presenta la obra y ubica algunas de sus aportaciones. Para el resto del libro realiza una crítica destructiva sin que hubiera un claro entendimiento de su parte en torno a las exposiciones de los autores, de manera que sus dichos se quedan en ocurrencias propias que en absoluto representan lo argumentado en la obra.

Por ejemplo, en la parte de la reseña dedicada a la primera sección hizo un abordaje razonable tratando de rescatar argumentos clave de su autor, Adolfo Bonilla. Lo mismo puede decirse del espacio dedicado al estudio de Ann Jefferson, el primer capítulo de la sección 2. Puede haber discrepancias al respecto, pero me parece claro que Gustavo Palma leyó y procuró comprender a estos autores.

Los problemas verdaderamente graves de esta reseña aparecen en las páginas dedicadas al resto de los ensayos, donde más que reseñar editorializa, falseando los estudios y asignándoles expresiones a los autores que no son de ellos, repetidamente poniendo frases entrecomilladas que son más bien de su autoría e inexistentes en los capítulos de este libro, distorsionando así el interés que los potenciales lectores pudieran tener del mismo.

Para el capítulo 3, sección 2, de Brian Connaughton, Palma asegura que el autor plantea que Rafael Carrera, el caudillo sobresaliente de esa época en Guatemala, se "construyó a sí mismo” y que según la opinión de Connaughton Guatemala requería de una "mano dura". Desde luego, estas expresiones son de Gustavo Palma, jamás del estudio tratado, tergiversando su razonamiento, pues el autor del texto partió de la perspectiva contraria, del escaso control sobre el territorio y población ejercida por los gobiernos de la época. Además, contrario a lo que afirma Palma relativa a una perspectiva formada únicamente a partir de opiniones de extranjeros de la época, recurrió adicionalmente a la correspondencia interna de gobierno, los 
dictámenes jurídicos de los fiscales de la Suprema Corte, y la correspondencia personal de Carrera para descifrar algunas de las cuestiones clave que estaban en juego. Nada de eso lo rescata Palma, que prefiere sus propias ideas. Todavía añade el reseñador: "Debería indagarse sobre el arraigo institucional estatal a nivel territorial”. Pero eso es justamente lo que el estudio de Connaughton pretende al utilizar numerosos expedientes del Ramo de Gobernación.

Además de Connaughton, el llamado "arraigo institucional” también fue abordado por Juan Carlos Sarazúa, en el capítulo 4 de la sección 3. Palma le acredita a Sarazúa la expresión el “'lugar' de Carrera como catalizador”, sin ser eso lo que escribió. También asigna a Sarazúa la expresión “'bisagra’ articuladora entre las agendas del poder económico capitalino y las de actores locales”, que igualmente es de Palma, no de Sarazúa. Sugiere que el autor apunta a "ver y leer” más allá del caudillo, lo cual es cierto, pero tampoco es la expresión utilizada por Sarazúa. Curiosamente, Palma deja de lado los planteamientos de Sarazúa en cuanto a la construcción del Estado de Guatemala. Posicionándose dentro de una reciente corriente historiográfica, Sarazúa hace énfasis en el claro vínculo entre militarización y fiscalización para el levantamiento del aparato estatal. Esto es seguramente lo más novedoso de lo expuesto por Sarazúa, y cuestiona seriamente la historiografía todavía dominante para el periodo.

Pasando al escrito de Moisés Ornelas, capítulo 5 de la sección 3, Gustavo Palma le acredita la expresión "prosperidad para los habitantes de la república”, en referencia al periodo de Carrera. Frase del reseñador usada en comillas hasta dos veces, pero sin aparecer jamás en el análisis de Ornelas. También falsamente entrecomilla que Ornelas admite la necesidad de "medir su verdadero impacto social” respecto de las disposiciones fiscales estudiadas. Según Palma la investigación de Ornelas es “técnica”, pero el autor no lo apunta textualmente así. Es desconcertante este uso repetido de palabras y frases entre comillas 
discordante con la obra reseñada. Además, Ornelas aborda más de una década de prácticas fiscales emergentes, en paulatina maduración, en Guatemala, incluso enfatizando desde el título el enfoque que problematiza lo hallado: “ ¿Estabilidad económica igual a gobernabilidad?” $\mathrm{Y}$, sin embargo, Palma afirma que "No destaca el acumulado de conocimiento de la administración hacendaria y financiera que-según la fuente consultada-fue exitosa; es decir, existía capacidad técnica y administrativa, punto importante que evidenciaría el estado de la administración pública, más allá de la figura del caudillo.” Bien, en el epílogo a su estudio, Moisés Ornelas escribe para despejar toda duda: "mi ensayo constató la puesta en marcha en Guatemala de una política hacendaria cuya prioridad era establecer un manejo profesional de las cuentas que incidiera en el arreglo del gasto público y, por consiguiente, que ayudara al saneamiento de las finanzas del erario al permitir el pago de la deuda interna y externa."

Al tratar la investigación de Leticia González, capítulo 6 de la sección 3, refiere Palma al sustento del Estado, en la época colonial y posteriormente, asegurando que según la autora esto "fue posible" por los impuestos cobrados a la población indígena. La autora nunca usó tal expresión, y más bien problematiza toda la dinámica de cobro y evasión de impuestos a nivel popular, situación extremadamente complicada pero clave para contemplar la construcción difícil de la institucionalidad que Palma parecía exigir de este libro, pero cuando los autores la abordamos, cambia el tema a cosas que al parecer son de mayor interés para él, como cuestiones de justicia social y trato entre distintos sectores de la población. Tales temáticas son extremadamente importantes, pero ¿por qué exigir abordajes de institucionalidad y luego esquivar las interpretaciones en torno a su difícil construcción? Atina, sin embargo, al afirmar que el estudio de Leticia González "Es un ejercicio analítico para aproximarse a explicar un momento histórico desde un proceso, y no desde la figura de un caudillo.” 
Palma precisa que la cuarta sección del libro tiene que ver con mujeres y vida cotidiana, asegurando a la vez que aborda los esfuerzos del Estado por "morigerar" las costumbres populares. Una vez más, ningún capítulo en el libro utiliza esa palabra de esa manera, pese a que la pone en comillas. Según Palma, el estudio de Lizeth Jiménez está dedicado a "lugares ocupados" en tiempos de guerra y muerte, pero la autora jamás usa esa expresión.

Más medular, en este caso, disputa Palma el recurso de Jiménez al pensamiento conceptual de Eric Hobsbawm, porque "Según Hobsbawm la agenda de los bandoleros no era social, política ni revolucionaria; eran campesinos que se resistían a la ley". Pero a la muerte de Eric Hobsbawn, el antropólogo mexicano Gilberto López y Rivas afirmó exactamente lo contrario:

La interpretación de Hobsbawm sobre el bandolerismo social rompe con la tradición historiográfica que considera como mero delincuente, un fuera de la ley, a todo participante en las luchas armadas contra el poder establecido, situando en un primer plano, en el campo de la investigación histórica, a movimientos sociales que los prejuicios ideológicos y sociales habían relegado al anonimato de los archivos policiacos, las páginas sensacionalistas de los periódicos, leyendas, relatos y cantos populares. Es por eso que la crítica de Hobsbawm de que bandoleros y salteadores de caminos preocupan a la policía, pero también debieran preocupar al historiador, es completamente justa. ${ }^{3}$

Palma afirma que quiere ver más énfasis en la agencia y razones de las mujeres y demás sectores populares. Pero Lizeth Jiménez concluye afirmando exactamente que eso fue lo que realizó: "En la mayoría de los casos, las mujeres se verán empujadas por el peso de las circunstancias extraordinarias que impone la

3 Gilberto López y Rivas, “El concepto de bandolerismo social, en Eric Hobsbawm”, La Jornada (viernes 12 oct. 2012). 
guerra, la violencia, a modificar sus conductas, sus acciones y sus prácticas. Algunas tendrán que enfrentarse a la ley, otras serán defensoras de sus familiares, hijos, esposos, padres. Unas cuantas correrán los riesgos de ser colaboradoras o enlaces entre los grupos alzados y sus seguidores.” La autora, en este ensayo, detalló múltiples casos de la actuación de las mujeres en ese sentido. Según Gustavo Palma el énfasis de Hobsbawn, y por extensión Jiménez, “niega actorías políticas construidas desde circunstancias específicas de vida”. ¿Será que leyó otro ensayo? Ella es clara en su argumentación.

En las palabras dedicadas al capítulo de Tania Sagastume, Palma asegura que es un estudio para abatir la "desviación social”, pero este término en absoluto fue escrito por la autora. Palma asienta que "Considero que más que reformar costumbres, el aseguramiento del orden social fue $-\mathrm{y}$ sigue siendo- una preocupación permanente del poder hegemónico." Pide Palma saber más de los beneficiarios de las medidas adoptadas. Pero la autora ha escrito con nitidez que "pretendían civilizar y compeler al trabajo”, y por ende los beneficiarios serían el gobierno y los propietarios. Además, el reseñador pasa por encima del hecho que la mirada de la autora es en torno a continuidades, $\mathrm{y}$ cambios, entre las llamadas Reformas Borbónicas, los gobiernos liberales y el periodo asociado con el caudillo Rafael Carrera, habitualmente considerado conservador.

Más adelante Sagastume afirma: "En todos los casos, los argumentos de las leyes apelaban a la necesidad de alejar a los indígenas de los malos ejemplos y los abusos, pero la lectura política de estas disposiciones también sugiere los intentos para reducir y controlar las reuniones de miembros de los sectores populares en espacios de diversión pública.” Añade además, pese a la duda de Gustavo Palma relativa a las verdaderas intenciones de reformar costumbres que "Durante el siglo xIx, la preocupación por la moral pública gradualmente dio paso a la necesidad de conservar el orden, el cumplimiento de las leyes y que todos se 
aplicaran al trabajo.” ¿Podría ser más clara la autora al respecto? Todavía más adelante, la autora aclara que las intenciones de las medidas referidas tenían un contenido moral, otro económico y otro más de control político. Contundente. Aún más:

Después de 1839, iban a mantener y afinar los controles, pero la preocupación por la moral pública va a dar paso a la necesidad de conservar el orden, el cumplimiento de las leyes y, sobre todo, que los miembros de los sectores populares se aplicaran a su trabajo. El contexto histórico y los argumentos de las leyes evidencian que el sistema necesitaba cada vez más brazos para trabajos en obras públicas y en la agricultura, por lo que las penas impuestas a los vagos, ociosos y mal entretenidos se concentraron en ponerlos a trabajar.

Dicho de otra manera, y con relación al pedido de Palma por colocar mayor énfasis en la institucionalidad del Estado, Tania Sagastume deja claro que la legislación abordada fue parte de la construcción de la hegemonía política, económica y sociocultural del gobierno conservador.

Para el capítulo 9 de la sección 5, la última del libro, Palma asegura que Francisco Rodolfo González Galeotti ofrece una "mirada local" para esta época, aunque el autor en ningún lugar usa esa expresión entrecomillada por Palma. Igual que en el caso de los dos primeros estudios de esta obra, Palma parece haberlo leído. Enhorabuena. Pero el trabajo de González Galeotti es mucho más que una mirada local. Aborda la enorme complejidad étnica, y política, de la región estudiada. Además, el autor nos sugiere la necesidad de ver la época más allá de la rebelión del oriente del país, donde la ira popular desde 1837 dio pie al eventual surgimiento del gobierno conservador para abatir las repudiadas reformas liberales, y contemplar más de la geografía humana, política popular y confrontaciones étnicas y sociales del periodo que dificultaban la construcción de una verdadera gobernabilidad. 
Finalmente, para atender al Coda escrito por el coordinador del libro, Gustavo Palma escribe lo siguiente respecto a la convocatoria expresada allí para repensar la historia del periodo estudiado a la luz de la historiografía actual. Es un: "Reto que, considero, requería transitar de lecturas centradas en personajes épicos al análisis de los grupos sociales; sus aportes, contradicciones y luchas por posicionar sus agendas en una etapa en que, se dice, se planteó la posibilidad de redefinir las bases del Estado luego de largos siglos de dominación colonial española."

Y, sin embargo, a través de su propia reseña, por más deficiente -como claramente lo es, señala que los autores de esta obra dedicaron sus investigaciones a justamente eso. Si hubiera dejado a un lado concepciones previas y fobias que subyacen apenas debajo de la superficie de su reseña, habría visto eso y hubiera realizado una reseña certera, más honesta y más orientadora para los lectores potenciales. ¿Será que lo que pretendió era exactamente lo contrario: distorsionar el contenido de los estudios, desviar la atención y menospreciar para asegurar que el libro careciera de lecturas críticas verdaderas, como debe tener una obra de esta índole?

Como expresó en una ocasión Mark Twain: "cada hombre tiene su opinión privada sobre un libro”. Pero agregó con clara intención: "Los hechos, o lo que un hombre cree que son hechos, son siempre encantadores. [...] Obtén tus datos primero, y ... luego puedes distorsionarlos tanto como quieras". ${ }^{4}$

\footnotetext{
4 “An Interview with Mark Twain”, en Rudyard Kipling, From Sea to Sea, and other Sketches. Letters of Travel, London, MacMillan and Co., 1900, vol. II, pp. 182-198, citas en 194 y 196-197: "every man has his private opinion about a book"; "[...] facts, or what a man believes to be facts, are always delightful.... Get your facts first, and ... then you can distort them as much as you please."
} 